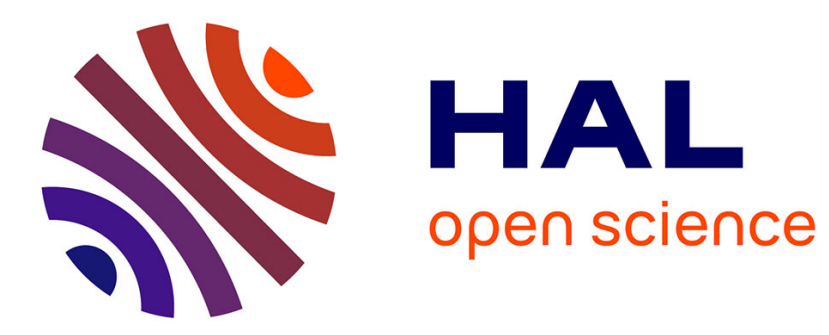

\title{
Geocoronal Lyman $\beta$ and Balmer $\alpha$ emissions measured during the Apollo 16 mission
}

R. R. Meier, G. R. Carruthers, T. L. Page, Anny Chantal Levasseur-Regourd

\section{To cite this version:}

R. R. Meier, G. R. Carruthers, T. L. Page, Anny Chantal Levasseur-Regourd. Geocoronal Lyman $\beta$ and Balmer $\alpha$ emissions measured during the Apollo 16 mission. Journal of Geophysical Research Space Physics, 1977, 82 (4), pp.737-739. 10.1029/JA082i004p00737 . insu-02263350

\section{HAL Id: insu-02263350 \\ https://hal-insu.archives-ouvertes.fr/insu-02263350}

Submitted on 12 Mar 2021

HAL is a multi-disciplinary open access archive for the deposit and dissemination of scientific research documents, whether they are published or not. The documents may come from teaching and research institutions in France or abroad, or from public or private research centers.
L'archive ouverte pluridisciplinaire HAL, est destinée au dépôt et à la diffusion de documents scientifiques de niveau recherche, publiés ou non, émanant des établissements d'enseignement et de recherche français ou étrangers, des laboratoires publics ou privés. 


\title{
Geocoronal Lyman $\beta$ and Balmer $\alpha$ Emissions Measured During the Apollo 16 Mission
}

\author{
R. R. Meier, G. R. Carruthers, and T. L. Page \\ E. O. Hulburt Center for Space Research, Naval Research Laboratory, Washington, D. C. 20375
}

A.-C. Levasseur-Regourd

Service d'Aéronomie du Centre National de la Recherche Scientifique, Verrières-le-Buisson, France

\begin{abstract}
Observations of the geocoronal Lyman $\beta$ emission rate were made with an electrographic camera/spectrograph during the Apollo 16 mission. These data along with geocoronal Balmer $\alpha$ airglow measurements obtained simultaneously from the D2A spacecraft are found to agree, to within experimental error, with an optically thick geocoronal hydrogen model.
\end{abstract}

\section{INTRODUCTION}

The first far ultraviolet spectrum of the terrestrial atmosphere in the wavelength range $500-1100 \dot{A}$ was made from the lunar surface during the Apollo 16 mission [Carruthers and Page, 1976a, $b$ ]. Among the spectral features observed was the Lyman $\beta(\mathrm{Ly} \beta)$ airglow line of atomic hydrogen at $1025.72 \AA$. Since this is the only spectrally pure measurement of this emission feature yet obtained, we present in this paper a detailed discussion of the Ly $\beta$ emission rate, comparing it with observations of the Balmer $\alpha(\mathrm{Ba} \alpha)$ emission rate at $6562.8 \AA$ which were obtained nearly simultaneously from the French D2A spacecraft. Both of these emissions result from excitation of geocoronal hydrogen to the $n=3$ quantum level by the solar Ly $\beta$ flux; subsequent transitions occur either to the ground state $(\mathrm{Ly} \beta)$ or to the $n=2$ level $(\mathrm{Ba} \alpha)$. The theoretical relationships between these emissions and the exciting solar flux are obtained by utilization of the theory of radiative transport in a spherical optically thick atmosphere.

\section{Data ANALysis}

The Ly $\beta$ observations were made with an electrographic Schmidt camera using film to record the images. (For details of the instrument, see Carruthers [1973], Carruthers and Page [1976a, b], and Carruthers et al. [1976].) The system could be utilized without additional optics for direct imagery or with an objective grating and electroformed grid collimator for spectra. A LiF corrector plate was used to improve the resolution of the camera for observations at wavelengths longward of $1050 \dot{A}$, but the instrument was used without a corrector (giving somewhat poorer spectral resolution) for spectrography at wavelengths below $1050 \AA$.

An isodensitometric plot of a terrestrial airglow spectrum was given in Figure 5 of Carruthers and Page [1976b]. The spectrograph 'slit' was oriented at an angle of about $25^{\circ}$ to the earth-sun line and slightly displaced from the center of the earth. A sketch of the observing geometry is shown in the insert of Figure 1. The observation was made on April 21, 1972 , when the sun-earth-moon angle was $105^{\circ}$. Because of the limited density resolution provided by isodensity contour plots of the type analyzed in their paper it was not possible to use the coarse density intervals shown in Figure 5 of Carruthers and Page [1976b] to measure very low densities in the spectra reliably and to remove the effects of backgrounds due to both

Copyright $\odot 1977$ by the American Geophysical Union. Paper number 6A0821. scattering in the camera and the rather low spectral resolution. Ghost lines and instrumental scattering of the intense Ly $\alpha$ line were not properly taken into account in the previous work. Therefore we resorted to the use of digitized printouts of the film density values to correct for these effects. Examples of such digitized printouts are given by Carruthers and Page [1976a]. Correcting for the above-mentioned backgrounds is a subjective exercise, so we conservatively took the uncertainties of the derived emission rates to be those resulting from use of the extreme limits of the allowable background. The result is rather large (but confident) error bars on the data. The degraded spatial resolution (perpendicular to the dispersion) in the correctorless mode yielded an uncertainty of about \pm 500 $\mathrm{km}$ at the earth in the position of a given density contour. Uncertainty in the location of the contours far from the earth is increased due to the low density levels on the film.

\section{LYMAN $\beta$ RESULTS}

Figure 1 shows the emission rate of $L y \beta$ as a function of distance along the spectrograph slit. The ' $O$ ' is referenced to the point of closest approach of the slit to the center of the earth. Data near the sunlit limb and the sunlit disc of the earth were deleted from the figure because of probable contamination from other thermospheric emission lines, such as O I $1027 \AA$. The altitude to which such contamination is significant can be estimated from the profiles (perpendicular to the dispersion) of other features due to $\mathrm{O}, \mathrm{N}_{2}$, etc., all of which (unlike hydrogen) have emission scale heights that are small in comparison to the camera resolution. Data given to the right of the break in Figure $1(2000$ to $-6000 \mathrm{~km})$ are from the portion of the slit covering the shadowed earth disc. Much of the emission there comes from sunlit hydrogen in the foreground, above the shadowed atmosphere. The calibration is given by Carruthers and Page [1976b] as $8.2 \times 10^{-4}$ density unit $(\mathrm{kR} \mathrm{s})^{-1}$ and is thought to be accurate to within $\pm 25 \%$.

The solid line in Figure 1 is the result of solving the optically thick spherical radiative transport equations for a three-level $\mathrm{H}$ atom. (See Meier [1969] for details of the formalism.) Resonant scattering of the solar Ly $\beta$ line is the excitation mechanism. The hydrogen density model was essentially the same as that used to analyze Ly $\alpha$ images [Carruthers et al., 1976] and other airglow observations [Meier and Mange, 1973]. For a model normalized to $3 \times 10^{7}$ atoms $\mathrm{cm}^{-3}$ at $100 \mathrm{~km}, T=1000$ $K$, and a critical satellite altitude of $R_{S C}=2.5$ (see Chamberlain [1963] for the definition of this parameter), the densities at 


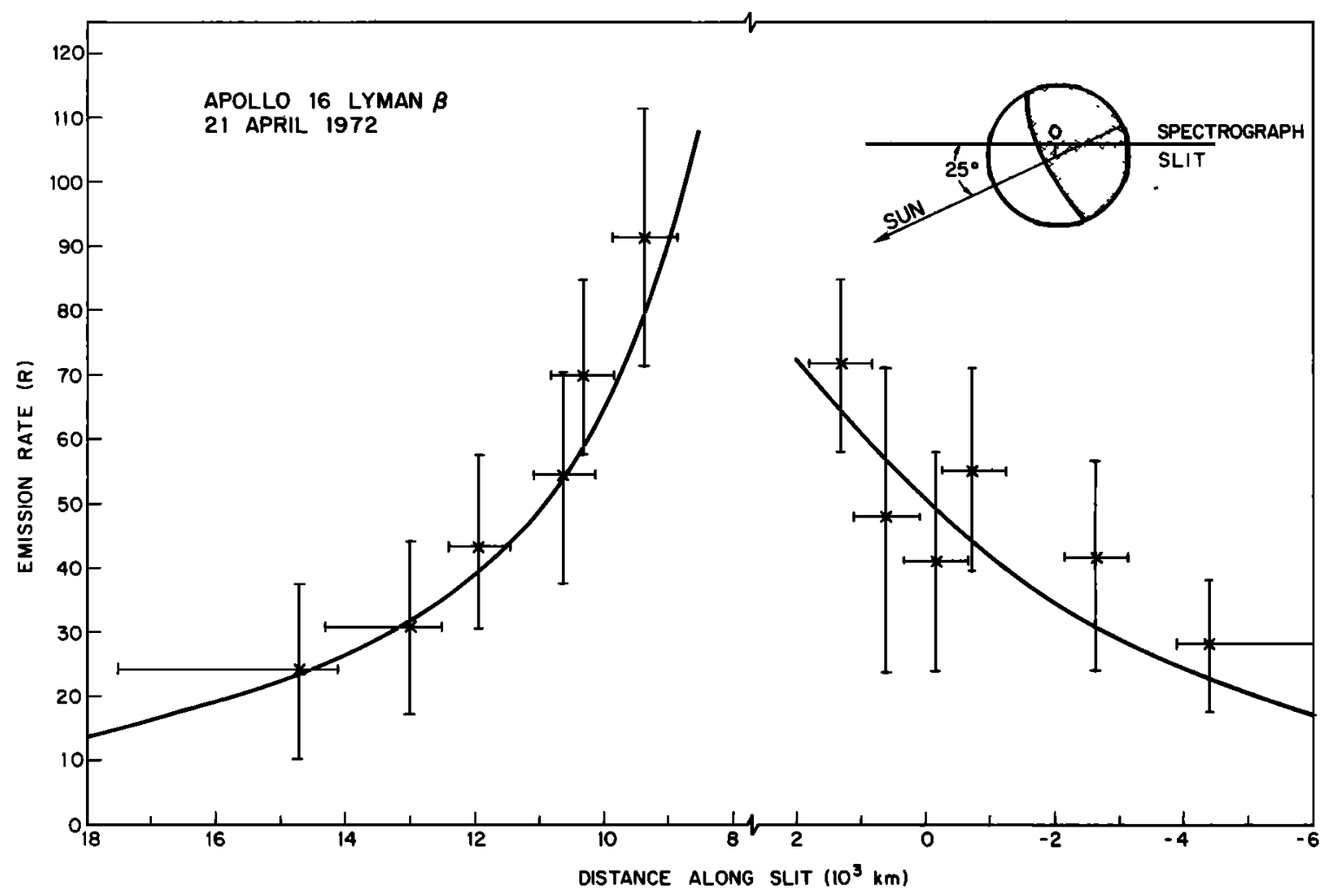

Fig. 1. Lyman $\beta$ emission rate as a function of distance along the spectrograph slit. The distance scale is measured from the closest approach of the slit to the center of the earth, indicated by $O$ in the insert at the upper right. Positive values are toward the sunlit hemisphere. The solid line is the result of multiple scattering calculations with an optically thick geocorona.

altitudes of 500,5000 , and $50,000 \mathrm{~km}$ are $80,000,4800$, and 27 atoms $\mathrm{cm}^{-3}$, respectively.

The agreement between the model and observation is good, in spite of the uncertainties in the data. The normalization in Figure 1 requires a solar Ly $\beta$ flux of $8.2 \times 10^{\circ}$ photons $\mathrm{cm}^{-2}$ $\mathbf{s}^{-1} \dot{A}^{-1}$ at the center of the line.

If the data were more precise, it would be of interest to compare the ratio of the $\operatorname{Ly} \alpha$ and $\operatorname{Ly} \beta$ emission rates. At high altitudes, where the geocorona is optically thin to both radiations ( $>20,000 \mathrm{~km}$ above the earth surface), the airglow ratio would yield the ratio of the solar emission line fluxes at the center of the line. At lower altitudes, where the geocorona becomes thick to Ly $\alpha$ but is still thin to $\operatorname{Ly} \beta$, their ratio would be a good measure of the multiple scattering component of the Ly $\alpha$ emission rate, which is related to the absolute value of the hydrogen column density. Although the Apollo 16 data were not sufficiently accurate to warrant a detailed analysis with multiple scattering models, it is of interest to note that the observed ratio of the Ly $\alpha$ [Carruthers et al., 1976] to Ly $\beta$ (using the crosses in Figure 1) ranged from near 200 at 15,000 $\mathrm{km}$ (along the spectrograph slit) to near 100 at $9000 \mathrm{~km}$. Unfortunately, the Ly $\beta$ feature was too weak to measure at altitudes where the Ly $\alpha$ is optically thin.

\section{Balmer $\alpha$ Results}

During the period of the Apollo 16 mission the French D2A spacecraft was operational. Among the experiments was an instrument designed to measure the geocoronal $\mathrm{Ba} \alpha$ emission rate [Levasseur and Blamont, 1973; Levasseur, 1976], which is also excited by the solar Ly $\beta$ line. Ba $\alpha$ data were obtained for several nights covering the Ly $\beta$ observation. Since the Ba $\alpha$ emission rate at night is very low ( $\sim 2 \mathrm{R}$ at midnight), all data obtained between April 17 and 25, 1972, were averaged for the same look directions in local coordinates to improve the statistics. A sample of these data is shown in Figure 2. The intensities were put into solar zenith angle (of the spacecraft) bins of $10^{\circ}$ width and plotted as a function of azimuth relative to the sun. The statistical uncertainty is less than $0.6 \mathrm{R}$ for each bin. The absolute calibration is thought to be accurate to within 10\% [Levasseur et al., 1976]. The line of sight of the instrument was normal to the spacecraft spin axis, which pointed toward the sun. Thus the angle of the line of sight to local zenith depended on azimuth and solar zenith angle [see Levasseur and Blamont, 1973]. The local zenith angle is plotted in the lower part of Figure 2. The solid lines in the data plot are the result of computations with the model of Figure 1 modified for the Ba $\alpha$ transition.

The solar Ly $\beta$ flux required to fit the Ba $\alpha$ data (a total of 22 averaged intensity points) was $4.9 \times 10^{\circ}$ photons $\mathrm{cm}^{-2} \mathrm{~s}^{-1} \dot{A}^{-1}$, a value which is only about $60 \%$ of that required by the $\operatorname{Ly} \beta$ airglow. The lower solar flux is more consistent with a recent analysis of a larger set of D2A and ground-based Ba $\alpha$ measurements [Levasseur et al., 1976]. Even though the Ly $\beta$ data were obtained on the sunlit geocorona and the $\mathrm{Ba} \alpha$ at night and both were compared with a spherically symmetric average hydrogen model, it is likely that the differences in the required solar flux can be attributed to data and calibration uncertainties.

Previous comparisons of the Ly $\beta$ and $\mathrm{Ba} \alpha$ geocoronal emissions were made by Weller et al. [1971]. While a higher solar Ly $\beta$ flux was required by their analysis $\left(1.75 \times 10^{10}\right.$ photons $\left.\mathrm{cm}^{-2} \mathrm{~s}^{-1} \dot{A}^{-1}\right)$, the broadband photometric mea- 


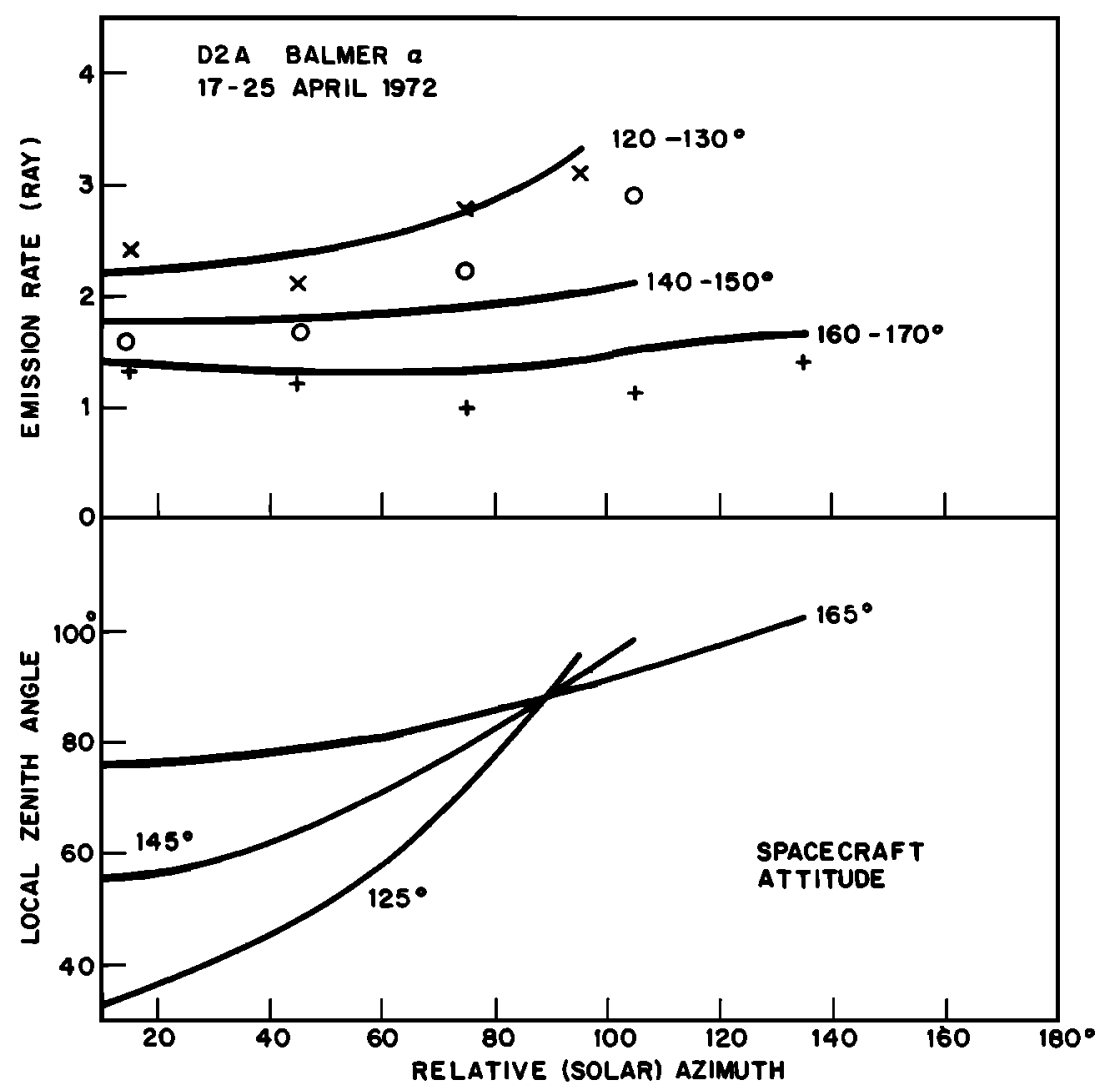

Fig. 2. Balmer $\alpha$ emission rate as a function of spacecraft line of sight altitude coordinates. The crosses, open circles, and pluses are for solar zenith angles in the range $120^{\circ}-130^{\circ}, 140^{\circ}-150^{\circ}$, and $160^{\circ}-170^{\circ}$, respectively. Spacecraft attitude coordinates are given for mean values of these solar zenith angle ranges.

surement of the Ly $\beta$ airglow appears to have contained additional emission features in the $800-$ to $1100-\AA$ wavelength interval. Furthermore, reanalysis of ground-based $\mathrm{Ba} \alpha$ observations requires a downward revision in the old airglow values [Levasseur et al., 1976]. Both of these considerations have the effect of reducing the deduced value of the solar flux. Thus the Ly $\beta$ airglow values computed by Meier [1974] should be revised downward in absolute value by a factor of $2-4$, since he used the high solar flux of Weller et al. [1971].

\section{SUMmary}

The first spectrally pure observation of the geocoronal hydrogen emission of Ly $\beta$ is reported here. Although uncertainties in the data lead to rather large error bars, the most likely values of the emission rate agree with those computed from 'standard' models of the geocorona. Comparison of the model with simultaneous measurements of the $\mathrm{Ba} \alpha$ emission rate from the D2A spacecraft also shows agreement to within experimental uncertainty. The solar flux required to produce the airglow emission rates is within $\pm 25 \%$ of $6.5 \times 10^{\circ}$ photons $\mathrm{cm}^{-2} \mathrm{~s}^{-1} \AA^{-1}$. The lower and upper limits are the best fits to the Ba $\alpha$ and Ly $\beta$ data, respectively.

Acknowledgment. The Editor thanks B. A. Tinsley for his assistance in evaluating this report.

\section{REFERENCES}

Carruthers, G. R., Apollo 16 far-ultraviolet camera/spectrograph: Instrument and operations, Appl. Opt., 12, 2501, 1973.
Carruthers, G. R., and T. L. Page, A pollo 16 far ultraviolet imagery of the polar auroras, tropical airglow belts, and general airglow, $J$. Geophys. Res., 81, 483, 1976 .

Carruthers, G. R., and T. L. Page, Apollo 16 far ultraviolet spectra of the terrestrial airglow, J. Geophys. Res., 81, 1683, 1976 b.

Carruthers, G. R., T. L. Page, and R. R. Meier, Apollo 16 Lyman alpha imagery of the hydrogen geocorona, J. Geophys. Res., 81, $1664,1976$.

Chamberlain, J. W., Planetary coronae and atmospheric evaporation, Planet. Space Sci., 11, 901, 1963.

Levasseur, A.-C., Observations atmosphériques et astronomiques au voisinage de $6563 \AA$ à bord du satellite D2A, thèse de doctorat d'état, Univ. Pierre et Marie Curie (Paris VI), Paris, 1976.

Levasseur, A.-C., and J. E. Blamont, Satellite observations of strong Balmer alpha atmospheric emissions around the magnetic equator, J. Geophys. Res., 78, 3881, 1973.

Levasseur, A.-C., R. R. Meier, and B. A. Tinsley, Resolution of the discrepancy between Balmer $\alpha$ emission rates, the solar Lyman $\beta$ flux, and models of geocoronal hydrogen concentration, J. Geophys. Res., 81, 5587, 1976.

Meier, R. R., Balmer alpha and Lyman beta in the hydrogen geocorona, J. Geophys. Res., 74, 3561, 1969.

Meier, R. R., A survey of the ultraviolet airglow from $1216 \AA$ A to 304 A, Ann. Geophys., 30, 91, 1974.

Meier, R. R., and P. Mange, Spatial and temporal variations in the Lyman-alpha airglow and related atomic hydrogen distributions, Planet. Space Sci., 21, 309, 1973.

Weller, C. S., R. R. Meier, and B. A. Tinsley, Simultaneous measurements of the hydrogen airglow emission of Lyman alpha, Lyman beta, and Balmer alpha, J. Geophys. Res., 76, 7734, 1971. 